\title{
Halogenated 1-Hydroxynaphthalene-2-Carboxanilides Affecting Photosynthetic Electron Transport in Photosystem II ${ }^{\dagger}$
}

\author{
Tomas Gonec ${ }^{1, *}$, Jiri Kos ${ }^{1,2}$, Matus Pesko ${ }^{3}$, Jana Dohanosova ${ }^{4}$, Michal Oravec ${ }^{5}$, Tibor Liptaj ${ }^{4}$, \\ Katarina Kralova ${ }^{6}$ and Josef Jampilek ${ }^{2, *}$ \\ 1 Department of Chemical Drugs, Faculty of Pharmacy, University of Veterinary and Pharmaceutical Sciences, \\ Palackeho 1, Brno 61242, Czech Republic; jurd@email.cz \\ 2 Department of Pharmaceutical Chemistry, Faculty of Pharmacy, Comenius University, Odbojarov 10, \\ Bratislava 83232, Slovakia \\ 3 Department of Environmental Ecology, Faculty of Natural Sciences, Comenius University, Ilkovicova 6, \\ Bratislava 84215, Slovakia; matus.pesko@gmail.com \\ 4 Central Laboratories, Faculty of Chemical and Food Technology, Slovak University of Technology in Bratislava, \\ Radlinskeho 9, Bratislava 81237, Slovakia; jana.dohanosova@stuba.sk (J.D.); tibor.liptaj@stuba.sk (T.L.) \\ 5 Global Change Research Institute CAS, Belidla 986/4a, 60300 Brno, Czech Republic; oravec.m@czechglobe.cz \\ 6 Institute of Chemistry, Faculty of Natural Sciences, Comenius University, Ilkovicova 6, Bratislava 84215, \\ Slovakia; kata.kralova@gmail.com \\ * Correspondence: t.gonec@seznam.cz (T.G.); josef.jampilek@gmail.com (J.J.); Tel.: +420-541-562-938 (T.G.) \\ + Preliminary results were presented at the 46th EuroCongress on Drug Synthesis and Analysis, Bratislava, \\ Slovakia, 5-8 September 2017 (paper P-61).
}

Received: 21 September 2017; Accepted: 9 October 2017; Published: 12 October 2017

\begin{abstract}
Series of seventeen new multihalogenated 1-hydroxynaphthalene-2-carboxanilides was prepared and characterized. All the compounds were tested for their activity related to the inhibition of photosynthetic electron transport (PET) in spinach (Spinacia oleracea L.) chloroplasts. 1-Hydroxy- $N$-phenylnaphthalene-2-carboxamides substituted in the anilide part by 3,5-dichloro-, 4-bromo-3-chloro-, 2,5-dibromo- and 3,4,5-trichloro atoms were the most potent PET inhibitors $\left(\mathrm{IC}_{50}=5.2,6.7,7.6\right.$ and $8.0 \mu \mathrm{M}$, respectively). The inhibitory activity of these compounds depends on the position and the type of halogen substituents, i.e., on lipophilicity and electronic properties of individual substituents of the anilide part of the molecule. Interactions of the studied compounds with chlorophyll $a$ and aromatic amino acids present in pigment-protein complexes mainly in PS II were documented by fluorescence spectroscopy. The section between $\mathrm{P}_{680}$ and plastoquinone $\mathrm{Q}_{B}$ in the PET chain occurring on the acceptor side of PS II can be suggested as the site of action of the compounds. The structure-activity relationships are discussed.
\end{abstract}

Keywords: hydroxynaphthalene-carboxamides; photosynthetic electron transport (PET) inhibition; spinach chloroplasts; structure-activity relationships

\section{Introduction}

In spite of the fact that various classes of herbicides are known and currently can be classified according to about 20 different modes of action [1], over $50 \%$ of marketed herbicides act by reversible binding to photosystem II (PS II) [2]. This effect results in the interruption of the photosynthetic electron transport (PET) [3-5]. PS II uses light energy to drive two chemical reactions: the oxidation of water and the reduction of plastoquinone. The following redox components of PS II participate in the transfer of electrons from $\mathrm{H}_{2} \mathrm{O}$ to the plastoquinone pool: water oxidizing manganese cluster $(\mathrm{Mn})_{4}$ and redox-active tyrosine $\left(\mathrm{Tyr}_{\mathrm{z}}\right)$ situated on the donor side of PS II, reaction centre chlorophyll (P680) 
and components situated on the acceptor side of PS II, namely pheophytin and two plastoquinone molecules, $\mathrm{Q}_{\mathrm{A}}$ and $\mathrm{Q}_{\mathrm{B}}$. While the plastoquinone molecule $\mathrm{Q}_{\mathrm{A}}$ acting as a one-electron acceptor is permanently bound to PS II, the plastoquinone molecule $\mathrm{Q}_{B}$ acting as a two-electron acceptor is loosely bound at the $\mathrm{Q}_{\mathrm{B}}$-site, and reduced plastoquinone unbinds from the reaction centre and diffuses in the hydrophobic core of the membrane, whereby $\mathrm{Q}_{B}$-binding site will be occupied by an oxidized plastoquinone molecule [6]. Herbicides belonging to photosystem (PS) II inhibitors inhibit photosynthetic electron transport (PET) by binding to the $\mathrm{Q}_{B}$-binding niche on the $\mathrm{D}_{1}$ protein of the PS II complex in chloroplast thylakoid membranes, which results in the inhibition of photosynthetic electron transport (PET) from $\mathrm{Q}_{\mathrm{A}}$ to $\mathrm{Q}_{\mathrm{B}}$, blockage of $\mathrm{CO}_{2}$ fixation and inhibition of ATP production. Many QSAR studies of PS II inhibitors with diverse chemical structures have emphasized the hydrophobic nature of the binding domain, with lipophilicity being the dominant determinant of Hill inhibition activity [7], e.g., the review paper of Lambreva et al. [8] presents a compendious overview of the recent improvements in the understanding of the structure and function of PS II donor side with a focus on the interactions of the plastoquinone cofactors with the surrounding environment and operational features. PET inhibitors in the functional dissection of the photosynthetic electron transport system were summarized in the review paper of Trebst [9], and a comprehensive overview of synthetic photosynthetic inhibitors and those based on natural products was published by Teixeira et al. [10].

Many herbicides acting as PET inhibitors in PS II containing an amide and/or a carbamate moiety, which is able to form hydrogen bonds between the $\mathrm{CONH} / \mathrm{NHCOO}$ group of amides/carbamates and the target proteins in photosynthetic centres of thylakoid membranes resulting in changes in protein conformation and finally in PET inhibition, have been shown to be the most potent type of this class of herbicides [11-13]. Both moieties can be substituted, and thus, their properties can be modified [14,15]. Amides are considered to be PS II inhibitors causing displacement of $Q_{B}$ from its binding pocket in the $\mathrm{D}_{1}$ protein [16], and halogen substituents were found to contribute to enhanced PET-inhibiting activity [10-12,17-23]. Our team has investigated effects of various amides [11,16,18-22,24-28] or carbamates [29-34] on biological systems, in particular, as anti-invasive agents for, a long time. In addition, some of previously published agents showed herbicidal properties associated with adverse effects on PS II [12,13,16,18-24,28,29,33].

In the context of the above-mentioned facts, new di- and trichlorinated and brominated 1-hydroxynaphthalene-2-carboxanilides were prepared and characterized and tested for their activity related to the PET inhibition in spinach (Spinacia oleracea L.) chloroplasts. The results were completed by seven recently published unsubstituted and monochlorinated and brominated derivatives [11], and the structure-activity relationships (SAR) of all the mentioned compounds are discussed.

\section{Results and Discussion}

\subsection{Chemistry}

The discussed anilides were synthesized using microwave-assisted synthesis as described in Gonec et al. [11]. All the studied compounds were prepared according to Scheme 1 . The condensation of 1-hydroxy-2-naphthoic acid and ring-substituted aniline using phosphorus trichloride in chlorobenzene gave a series of twenty-four 1-hydroxynaphthalene-2-carboxanilides 1-24.

Physicochemical descriptors-lipophilicity of the compounds expressed as $\log P$ and electronic $\sigma$ parameters of individual anilides-were calculated for all the investigated compounds by means of the ACD/Percepta ver. 2012 program, see Table 1. Log $P$ values ranged from 4.52 (unsubstituted anilide 1) to $6.34(2,4,6-\mathrm{Br}$ substituted compound 20). In general, it can be stated that the lipophilicity is rather high; nevertheless, the recommended $\log P$ value for agrochemicals is $\leq 5$ [35]. Beside unsubstituted compound 1, monohalogenated anilides 2-7 expressed the lowest lipophilicity, while trisubstituted derivatives 14-16 and $\mathbf{2 0}$ possessed the highest $\log P$ values, and in general brominated compounds showed higher lipophilicity than chlorinated. For individual $N$-aryl parts of the anilides also electronic properties expressed as electronic $\sigma$ constants of the whole substituted phenyl ring were predicted; they ranged from 0.60 (compound $\mathbf{1})$ to $1.56(2,4,5-\mathrm{Cl}$ substituted derivative 16). 


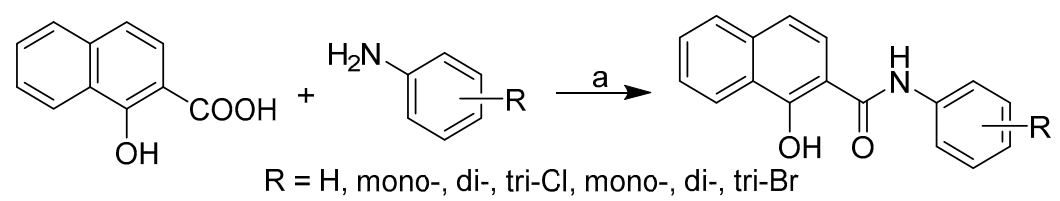

Scheme 1. Synthesis of ring-substituted 1-hydroxynaphthalene-2-carboxanilides 1-24. Reagents and conditions: (a) $\mathrm{PCl}_{3}$, chlorobenzene, microwave synthesis.

Table 1. Structure of ring-substituted 1-hydroxynaphthalene-2-carboxanilides 1-24, calculated values of $\log P$, electronic $\sigma$ parameters of anilide $(\mathrm{Ar})$ substituents and $\mathrm{IC}_{50}[\mu \mathrm{M}]$ values related to photosynthetic electron transport (PET) inhibition in spinach chloroplasts in comparison with 3-(3,4-dichlorophenyl)-1,1-dimethylurea (DCMU) standard.



\begin{tabular}{|c|c|c|c|c|}
\hline Comp. & $\mathbf{R}$ & $\log P^{a}$ & $\sigma_{(\mathrm{Ar})}{ }^{a}$ & PET Inhibition $\mathrm{IC}_{50}[\mu \mathrm{M}]$ \\
\hline $1^{b}$ & $\mathrm{H}$ & 4.52 & 0.60 & 31.3 \\
\hline $2^{b}$ & $2-\mathrm{Cl}$ & 5.02 & 1.05 & 29.4 \\
\hline $3^{b}$ & $3-\mathrm{Cl}$ & 5.25 & 0.85 & 7.9 \\
\hline $4^{b}$ & $4-\mathrm{Cl}$ & 5.24 & 0.75 & 10.8 \\
\hline $5^{b}$ & $2-\mathrm{Br}$ & 5.06 & 0.97 & 61.9 \\
\hline $6^{b}$ & $3-\mathrm{Br}$ & 5.39 & 0.86 & 8.2 \\
\hline $7^{b}$ & $4-\mathrm{Br}$ & 5.28 & 0.74 & 9.6 \\
\hline 8 & $2,3-\mathrm{Cl}$ & 5.76 & 1.22 & 235.3 \\
\hline 9 & $2,4-\mathrm{Cl}$ & 5.78 & 1.12 & 531.0 \\
\hline 10 & $2,5-\mathrm{Cl}$ & 5.82 & 1.22 & 59.6 \\
\hline 11 & $2,6-\mathrm{Cl}$ & 5.52 & 1.33 & 100.5 \\
\hline 12 & $3,4-\mathrm{Cl}$ & 5.99 & 1.19 & 13.3 \\
\hline 13 & $3,5-\mathrm{Cl}$ & 6.01 & 1.11 & 5.2 \\
\hline 14 & $2,4,5-\mathrm{Cl}$ & 6.31 & 1.56 & 153.2 \\
\hline 15 & $2,4,6-\mathrm{Cl}$ & 6.15 & 1.48 & 10.6 \\
\hline 16 & $3,4,5-\mathrm{Cl}$ & 6.28 & 1.46 & 8.0 \\
\hline 17 & $2,4-\mathrm{Br}$ & 5.90 & 1.11 & 505.1 \\
\hline 18 & $2,5-\mathrm{Br}$ & 5.81 & 1.23 & 7.6 \\
\hline 19 & $2,6-\mathrm{Br}$ & 5.67 & 1.33 & 78.0 \\
\hline 20 & $2,4,6-\mathrm{Br}$ & 6.34 & 1.47 & 45.0 \\
\hline 21 & $2-\mathrm{Br}-4-\mathrm{Cl}$ & 5.80 & 1.12 & 277.5 \\
\hline 22 & $4-\mathrm{Br}-2-\mathrm{Cl}$ & 5.88 & 1.11 & 171.0 \\
\hline 23 & $5-\mathrm{Br}-2-\mathrm{Cl}$ & 5.82 & 1.23 & 60.3 \\
\hline 24 & 4-Br-3-Cl & 6.02 & 1.19 & 6.7 \\
\hline DCMU & - & - & - & 1.9 \\
\hline
\end{tabular}

in Gonec et al. [11]

\subsection{Inhibition of Photosynthetic Electron Transport (PET) in Spinach Chloroplasts}

In this contribution, variously chlorinated and brominated anilides and their PET-inhibiting activity are discussed. The PET-inhibiting activity was expressed by $\mathrm{IC}_{50}$ value (compound concentration causing $50 \%$ inhibition of PET), see Table 1 . The activity of the studied compounds was compared with diuron (3-(3,4-dichlorophenyl)-1,1-dimethylurea; DCMU), a commercial PET inhibitor. The obtained activity showed a wide range of PET inhibition, from $5.2 \mu \mathrm{M}$ (compound $13, \mathrm{R}=3,5-\mathrm{Cl}$ ) to $531 \mu \mathrm{M}$ (compound $9, \mathrm{R}=2,4-\mathrm{Cl}$ ). In general, it can be stated that chlorinated derivatives demonstrated higher potency than bromine derivatives. 2,4-Disubstituted compounds, i.e., $9(\mathrm{R}=2,4-\mathrm{Cl}), 17(\mathrm{R}=2,4-\mathrm{Br}), 21(\mathrm{R}=2-\mathrm{Br}-4-\mathrm{Cl}), 22(\mathrm{R}=2-\mathrm{Cl}-4-\mathrm{Br})$, and one 2,3-disubstituted compound 
$8(\mathrm{R}=2,3-\mathrm{Cl})$ showed limited aqueous solubility compared with 2,5-, 2,6-, 3,4- and 3,5-disubstituted derivatives or the rest of compounds. Therefore, these compounds are excluded from the SAR discussion and this is illustrated on the figures by empty symbols, see below. It should be noted that the highest potency with $\mathrm{IC}_{50}=5.2 \mu \mathrm{M}$ was shown by symmetrically disubstituted compound $13(\mathrm{R}=3,5-\mathrm{Cl})$, while the trisubstituted analogue-compound $16(\mathrm{R}=3,4,5-\mathrm{Cl})$ —expressed already PET-inhibiting activity $\mathrm{IC}_{50}=8.0 \mu \mathrm{M}$, and mono-chlorinated derivatives 3 and 4 showed $\mathrm{IC}_{50}$ of $7.9 \mu \mathrm{M}$ $(\mathrm{R}=3-\mathrm{Cl})$ and $10.8 \mu \mathrm{M}(\mathrm{R}=4-\mathrm{Cl})$, respectively. Thus, it seems that physicochemical properties resulting from $\mathrm{C}_{(3,5)}^{\prime}$ position of both chlorine atoms are preferable.

The dependences of the PET-inhibiting activity on the lipophilicity of the discussed mono-, diand tri- $N$-aryl substituted anilides are shown in Figure 1A. Similar trends for mono- and disubstituted compounds can be observed-the PET-inhibiting activity increases with increasing lipophilicity, while an opposite trend can be found for trisubstituted derivatives. Thus, it seems that for PET-inhibiting activity, a lipophilicity optimum is in the range of $\log P$ from 6.01 (compound 13) to 6.28 (compound 16), see Figure 1A. It is important to note that a PET inhibition depends also on the electronic effects of individual substituents within series of different PET inhibitors [11,12,21-23]. The dependences of the PET-inhibiting activity on the electron $\sigma$ parameters of the whole $N$-aryl part of individual anilides are illustrated in Figure 1B. Practically the same trends can be found for mono-, di- and trisubstituted derivatives; the PET inhibition sharply decreases with the increasing electron-withdrawing effect of individual anilides (i.e., with high amide bond electron-deficiency) as follows: compound $3(\mathrm{R}=3-\mathrm{Cl}$, $\left.\sigma=0.85, \mathrm{IC}_{50}=7.9 \mu \mathrm{M}\right)>>>$ compound $5\left(\mathrm{R}=2-\mathrm{Br}, \sigma=0.97, \mathrm{IC}_{50}=61.9 \mu \mathrm{M}\right)$; compound $13(\mathrm{R}=3,5-\mathrm{Cl}$, $\left.\sigma=1.11, \mathrm{IC}_{50}=5.2 \mu \mathrm{M}\right)>>>$ compound $11\left(\mathrm{R}=2,6-\mathrm{Cl}, \sigma=1.33, \mathrm{IC}_{50}=100.5 \mu \mathrm{M}\right)$; compound 16 $\left(\mathrm{R}=3,4,5-\mathrm{Cl}, \sigma=1.46, \mathrm{IC}_{50}=8.0 \mu \mathrm{M}\right)>>>$ compound $14\left(\mathrm{R}=2,4,5-\mathrm{Cl}, \sigma=1.56, \mathrm{IC}_{50}=153.2 \mu \mathrm{M}\right)$.
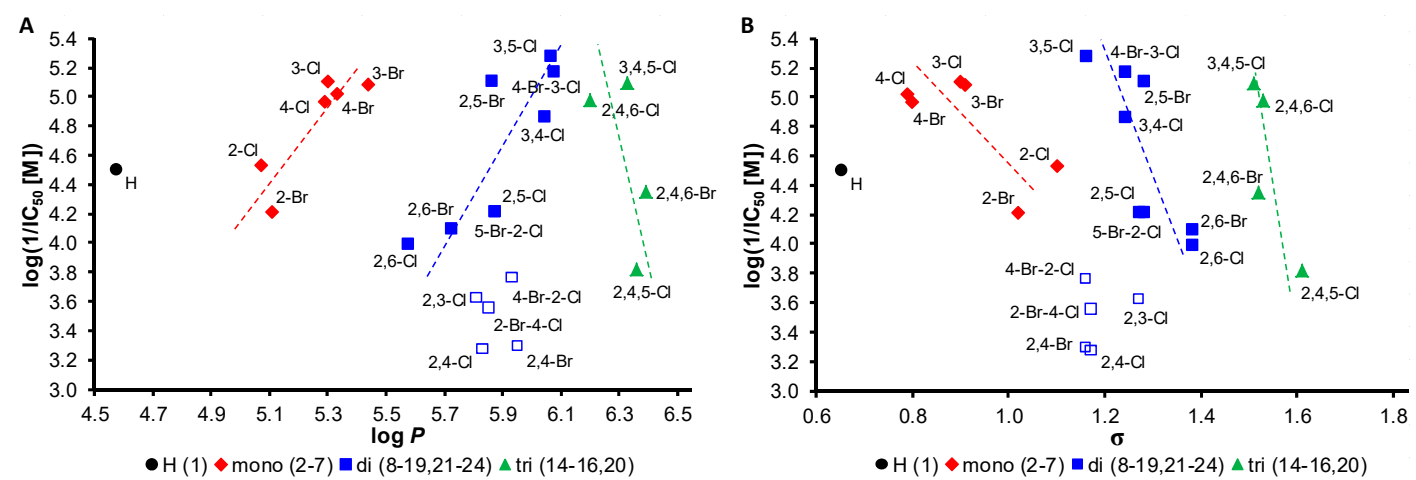

Figure 1. Dependence of PET-inhibiting activity $\log \left(1 / \mathrm{IC}_{50}[\mathrm{M}]\right)$ of all discussed compounds 1-24 in spinach chloroplasts on lipophilicity expressed as $\log P(\mathbf{A})$ and electronic $\sigma$ parameters of whole $\mathrm{N}$-aryl part of individual anilides (B). Empty squares are not involved in SAR discussion.

1,5-Diphenylcarbazide (DPC), an efficient artificial electron donor to the inactive oxygen-evolving complex (OEC) of PS II acting in Z/D intermediate situated on the donor side of PS II can be used for a more precise determination of the site of action of the tested compounds in the PET chain [36-38]. This intermediate ensures electron transport from the OEC to the core of PS II (P680) [39]. Consequently, an addition of DPC can completely restore the activity of chloroplasts, which was inhibited by PET inhibitors acting on the donor side of PS II. However, the addition of DPC to chloroplasts, the activity of which was inhibited by tested compounds approximately up to $80 \%$ (i.e., corresponding to $20 \%$ activity of the control), caused gradual PET restoration with increasing DPC concentration, and complete activity restoration was obtained only with 12-fold higher DPC concentration compared to the concentration of the applied PET inhibitor. Based on this finding, it could be suggested that the site of inhibitory action of the halogenated 1-hydroxynaphthalene-2-carboxanilides is situated on the acceptor side of PS II, at the section between P680 (primary donor of PS II) and $\mathrm{Q}_{\mathrm{B}}$. The complete restoration of PET using DPC concentration exceeding that of the applied 
inhibitor by more than one order of magnitude indicates that, due to direct interaction of DPC with the herbicide binding niche, these PET inhibitors were displaced from their binding site, as was similarly shown for atrazine [40] or metribuzin [41]. The plastoquinone $\mathrm{Q}_{\mathrm{B}}$ at the acceptor side of PS II was found to be the site of inhibitory action of $N$-benzylpyrazine-2-carboxamides [42] and $N$-alkoxyphenyl-3-hydroxy-naphthalene-2-carboxamides [16] as well as ring-substituted salicylanilides, carbamoylphenyl-carbamates [29] and 8-hydroxyquinoline-2-carboxamides [23].

In isolated chloroplasts, the interaction of a PET inhibitor with chlorophyll $a$ (Chla) molecules situated in pigment-protein complexes in PS II is reflected in changes of the intensity of Chla fluorescence emission band at $686 \mathrm{~nm}$. A gradual decrease of the intensity of this Chl $a$ emission band at $686 \mathrm{~nm}$ with the increasing concentration of $N$-(5-bromo-2-chlorophenyl)-1-hydroxynaphthalene2-carboxamide (23) (Figure 2A) indicates increased perturbation of Chla-protein complexes in the thylakoid membrane [43] by this PET inhibitor. A similar Chla fluorescence decrease in spinach chloroplasts was observed previously for several PET inhibitors, such as substituted benzanilides [44], $N$-benzylpyrazine-2-carboxamides [42], 3,5-dibromo-2-hydroxy- $N$-phenylbenzamides [45], ringsubstituted 8-hydroxyquinoline-2-carboxamides [23], 3-hydroxynaphthalene-2-carboxamides [21] and 2-alkylpyridine-4-carboxylic acids [46].

In addition, the interaction of PET inhibitors with aromatic amino acids (AAA), mainly tryptophan and tyrosine, occurring in photosynthetic proteins situated mainly in PS II can be investigated using the fluorescence method. In the presence of the tested halogenated 1-hydroxynaphthalene-2-carboxanilides, quenching of AAA fluorescence at $334 \mathrm{~nm}$ was observed, which showed an increase with increasing concentration of PET inhibitors, which is presented in Figure 2B for the model compound 23. It could be assumed that the decrease in fluorescence is caused by a change in the environment of AAAs upon interaction with the tested compounds. The quenching of the AAA fluorescence in the presence of various PET inhibitors such as ring-substituted 8-hydroxyquinoline-2-carboxamides [23], 5-bromo- and 3,5-dibromo-2-hydroxy$N$-phenylbenzamides [45], N-substituted 5-amino-6-methylpyrazine-2,3-dicarbonitriles [47] and 2-substituted 6-fluoro-benzothiazoles [48] was observed previously.
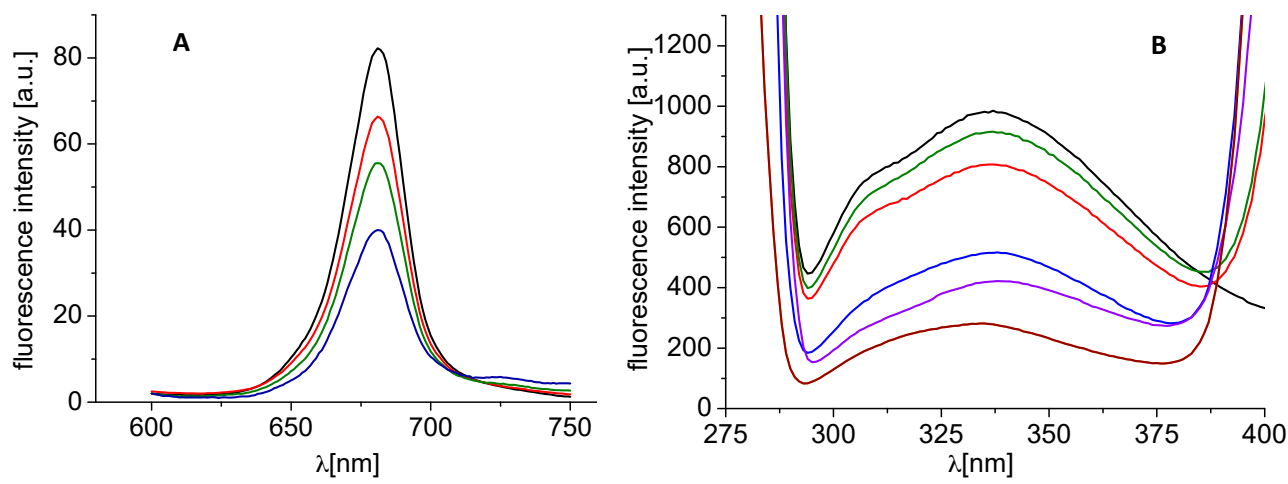

Figure 2. Fluorescence emission spectra of chlorophyll $a$ in untreated spinach chloroplasts in presence of compound 23 ( $\mathrm{R}=5-\mathrm{Br}-2-\mathrm{Cl} ; \mathrm{c}=0,56,220$, and $431 \mu \mathrm{M}$; curves from top to bottom); excitation wave length $\lambda_{\mathrm{ex}}=436 \mathrm{~nm}$, chlorophyll concentration $10 \mathrm{mg} / \mathrm{L}(\mathrm{A})$ and fluorescence emission spectra of aromatic amino acids in suspension of spinach chloroplasts without and with compound $\mathbf{2 3}(\mathrm{c}=0,5.9$, 11.8, 23.6, 29.5 and $47.2 \mu \mathrm{M}$; curves from top to bottom); excitation wave length $\lambda=275 \mathrm{~nm}$, chlorophyll concentration $10 \mathrm{mg} / \mathrm{L}(\mathbf{B})$.

\section{Materials and Methods}

\subsection{General Information}

All reagents were purchased from Merck (Sigma-Aldrich, St. Louis, MO, USA) and Alfa (Alfa-Aesar, Ward Hill, MA, USA). Reactions were performed using a CEM Discover SP microwave 
reactor (CEM, Matthews, NC, USA). The melting points were determined on a Kofler hot-plate apparatus HMK (Franz Kustner Nacht KG, Dresden, Germany) and are uncorrected. Infrared (IR) spectra were recorded on a Smart MIRacle ${ }^{\mathrm{TM}}$ ATR ZnSe for Nicolet ${ }^{\mathrm{TM}}$ Impact 410 Fourier-transform IR spectrometer (Thermo Scientific, West Palm Beach, FL, USA). The spectra were obtained by the accumulation of 256 scans with $2 \mathrm{~cm}^{-1}$ resolution in the region of $4000-650 \mathrm{~cm}^{-1}$. All ${ }^{1} \mathrm{H}-$ and ${ }^{13} \mathrm{C}-\mathrm{NMR}$ spectra were recorded on an Agilent VNMRS $600 \mathrm{MHz}$ system $\left(600 \mathrm{MHz}\right.$ for ${ }^{1} \mathrm{H}$ and $150 \mathrm{MHz}$ for ${ }^{13} \mathrm{C}$, Agilent Technologies, Santa Clara, CA, USA) in dimethyl sulfoxide- $d_{6}$ (DMSO- $d_{6}$ ). ${ }^{1} \mathrm{H}$ and ${ }^{13} \mathrm{C}$ chemical shifts $(\delta)$ are reported in ppm. The prepared compounds were analysed by an ultra-high performance liquid chromatograph (Dionex UltiMate ${ }^{\circledR} 3000$ Liquid Chromatography Systems, Thermo Scientific) coupled with a diode-array detector (DAD, Thermo Scientific) and a high resolution Hybrid Ion Trap-Orbitrap XL ${ }^{\mathrm{TM}}$ Fourier transform mass spectrometer (HRMS, Thermo Scientific). A chromatographic column Hypersil Gold (Thermo Scientific), C18 $3 \mu \mathrm{m}, 2.1 \mathrm{~mm} \times 50 \mathrm{~mm}$, was used. The mixture of MeCN-HPLC grade (20.0\%) and $\mathrm{H}_{2} \mathrm{O}-\mathrm{HPLC}$ grade with $0.1 \% \mathrm{AcOH}(80.0 \%)$ was used as a mobile phase. The total flow of the column was $0.3 \mathrm{~mL} / \mathrm{min}$; the column temperature was $30^{\circ} \mathrm{C}$; and the time of analysis was $10 \mathrm{~min}$. The records were evaluated from the DAD and HRMS-Orbitrap. The wavelengths of 210, 254, 272, 274, $331 \mathrm{~nm}$ and 190-800 nm were monitored. The peaks in the chromatogram of the solvent (blank) were deducted from the peaks in the chromatogram of the sample solution. The purity of individual compounds was determined from the area peaks in the chromatogram of the sample solution at wavelength of $210 \mathrm{~nm}$. MS and MS ${ }^{\mathrm{n}}$ were performed using the HRMS-Orbitrap equipped with a HESI II (heated electrospray ionization) source.

\subsection{Synthesis}

General Procedure for Synthesis of N-(substituted phenyl)-1-hydroxynaphthalene-2-carboxamides 1-24

1-Hydroxynaphthalene-2-carboxylic acid $(5.3 \mathrm{mmol})$ and the corresponding substituted aniline ( $5.3 \mathrm{mmol}$ ) were suspended in $30 \mathrm{~mL}$ of dry chlorobenzene. Phosphorous trichloride $(2.65 \mathrm{mmol})$ was added dropwise, and the reacting mixture was heated in the microwave reactor at maximal allowed power $500 \mathrm{~W}$ and $130{ }^{\circ} \mathrm{C}$, using infrared flask-surface control of temperature, for $15 \mathrm{~min}$. The solvent was evaporated under reduced pressure, the solid residue washed with $2 \mathrm{M} \mathrm{HCl}$, and the crude product was recrystallized from aqueous ethanol. All the studied compounds are presented in Table 1.

Described anilides 1-7 were characterized recently by Gonec et al. [11].

N-(2,3-Dichlorophenyl)-1-hydroxynaphthalene-2-carboxamide (8). Yield 59\%; m.p. 199-201 ${ }^{\circ} \mathrm{C}$; HPLC purity 98.32\%; IR ( $\mathrm{cm}^{-1}$ ): 3418, 1634, 1580, 1532, 1504, 1450, 1408, 1390, 1358, 1327, 1302, 1271, 1243, 1205, 1190, 1150, 1089, 1044, 878, 824, 799, 779, 770, 755, 738, 722, 700; ${ }^{1} \mathrm{H}-\mathrm{NMR}$ (DMSO- $d_{6}$ ), $\delta: 13.67$ (br. s, $1 \mathrm{H}), 10.84(\mathrm{~s}, 1 \mathrm{H}), 8.32(\mathrm{~d}, 1 \mathrm{H}, J=7.8 \mathrm{~Hz}), 8.08(\mathrm{~d}, 1 \mathrm{H}, J=8.7 \mathrm{~Hz}), 7.93(\mathrm{~d}, 1 \mathrm{H}, J=8.2 \mathrm{~Hz}), 7.66-7.72(\mathrm{~m}$, $2 \mathrm{H}), 7.63(\mathrm{dd}, 1 \mathrm{H}, J=8.2, J=1.4 \mathrm{~Hz}), 7.60(\mathrm{ddd}, 1 \mathrm{H}, J=8.2, J=6.9, J=1.4 \mathrm{~Hz}), 7.50(\mathrm{~d}, 1 \mathrm{H}, J=1.2 \mathrm{~Hz})$, $7.47(\mathrm{t}, 1 \mathrm{H}, J=7.8 \mathrm{~Hz}) ;{ }^{13} \mathrm{C}-\mathrm{NMR}$ (DMSO- $\left.d_{6}\right), \delta: 169.22,159.37,136.23,136.14,132.06,129.24,128.49$, 128.44, 128.26, 127.61, 127.27, 126.06, 124.66, 123.23, 123.12, 118.41, 107.61; HR-MS: [M - H] ${ }^{+}$calculated $330.00831 \mathrm{~m} / \mathrm{z}$, found $330.01007 \mathrm{~m} / \mathrm{z}$.

N-(2,4-Dichlorophenyl)-1-hydroxynaphthalene-2-carboxamide (9). Yield 76\%; m.p. 181-184 ${ }^{\circ} \mathrm{C}$; HPLC purity 98.45\%; IR ( $\left.\mathrm{cm}^{-1}\right)$ : 3422, 1629, 1580, 1524, 1503, 1472, 1456, 1391, 1331, 1300, 1243, 1211, 1098, 867, 818, 796, 787, 761, 715; ${ }^{1} \mathrm{H}-\mathrm{NMR}$ (DMSO- $d_{6}$ ), $\delta: 13.67$ (br. s, $\left.1 \mathrm{H}\right), 10.74$ (s, $\left.1 \mathrm{H}\right), 8.32$ (d, $\left.1 \mathrm{H}, J=8.2 \mathrm{~Hz}\right), 8.08$ $(\mathrm{d}, 1 \mathrm{H}, J=8.7 \mathrm{~Hz}), 7.93(\mathrm{~d}, 1 \mathrm{H}, J=8.2 \mathrm{~Hz}), 7.79(\mathrm{~d}, 1 \mathrm{H}, J=2.3 \mathrm{~Hz}), 7.75(\mathrm{~d}, 1 \mathrm{H}, J=8.7 \mathrm{~Hz}), 7.68(\mathrm{ddd}$, $1 \mathrm{H}, J=8.1, J=7.0, J=0.9 \mathrm{~Hz}), 7.59(\mathrm{ddd}, 1 \mathrm{H}, J=8.2, J=6.9, J=1.4 \mathrm{~Hz}), 7.53(\mathrm{dd}, 1 \mathrm{H}, J=8.7, J=2.3 \mathrm{~Hz})$, $7.49(\mathrm{~d}, 1 \mathrm{H}, J=8.7 \mathrm{~Hz}) ;{ }^{13} \mathrm{C}-\mathrm{NMR}$ (DMSO- $\left.d_{6}\right), \delta: 169.17,159.31,136.13,133.40,131.40,130.70,129.70$, 129.22, 129.16, 127.87, 127.60, 126.04, 124.66, 123.24, 123.11, 118.40, 107.67; HR-MS: [M - H] ${ }^{+}$calculated $330.00831 \mathrm{~m} / \mathrm{z}$, found $330.01007 \mathrm{~m} / \mathrm{z}$.

N-(2,5-Dichlorophenyl)-1-hydroxynaphthalene-2-carboxamide (10). Yield 69\%; m.p. 194-196 ${ }^{\circ} \mathrm{C}$; HPLC purity $99.08 \%$; IR $\left(\mathrm{cm}^{-1}\right)$ : 3418, 1632, 1583, 1525, 1502, 1471, 1407, 1392, 1320, 1280, 1239, 1209, 1151, 
1089, 1047, 916, 876, 806, 786, 757, 725; ${ }^{1} \mathrm{H}-\mathrm{NMR}$ (DMSO-d $\left.d_{6}\right), \delta: 13.47(\mathrm{~s}, 1 \mathrm{H}), 10.83(\mathrm{~s}, 1 \mathrm{H}), 8.33$ (d, $1 \mathrm{H}, J=8.6 \mathrm{~Hz}), 8.05-8.10(\mathrm{~m}, 1 \mathrm{H}), 7.95(\mathrm{~d}, 1 \mathrm{H}, J=2.6 \mathrm{~Hz}), 7.93(\mathrm{~d}, 1 \mathrm{H}, J=8.2 \mathrm{~Hz}), 7.68(\mathrm{ddd}, 1 \mathrm{H}$, $J=8.2, J=6.9, J=1.3 \mathrm{~Hz}), 7.65(\mathrm{~d}, 1 \mathrm{H}, J=8.9 \mathrm{~Hz}), 7.60(\mathrm{ddd}, 1 \mathrm{H}, J=8.3, J=7.0, J=1.2 \mathrm{~Hz}), 7.51$ $(\mathrm{d}, 1 \mathrm{H}, J=8.9 \mathrm{~Hz}), 7.42(\mathrm{dd}, 1 \mathrm{H}, J=8.7, J=2.5 \mathrm{~Hz}) ;{ }^{13} \mathrm{C}-\mathrm{NMR}$ (DMSO- $\left.d_{6}\right), \delta: 168.70,158.81,136.15$, 135.64, 131.69, 130.92, 129.17, 127.67, 127.61, 127.40, 127.38, 126.03, 124.71, 123.48, 123.13, 118.58, 108.25; HR-MS: $[\mathrm{M}-\mathrm{H}]^{+}$calculated $330.00831 \mathrm{~m} / \mathrm{z}$, found $330.00986 \mathrm{~m} / \mathrm{z}$.

N-(2,6-Dichlorophenyl)-1-hydroxynaphthalene-2-carboxamide (11). Yield 59\%; m.p. 199-202 ${ }^{\circ} \mathrm{C}$; HPLC purity $98.82 \%$; IR $\left(\mathrm{cm}^{-1}\right)$ : 3309, 1635, 1616, 1596, 1568, 1529, 1499, 1448, 1435, 1383, 1331, 1283, 1252, $1209,944,796,766,721,701 ;{ }^{1} \mathrm{H}-\mathrm{NMR}$ (DMSO- $\left.d_{6}\right), \delta: 13.85(\mathrm{~s}, 1 \mathrm{H}), 10.76(\mathrm{~s}, 1 \mathrm{H}), 8.28-8.33(\mathrm{~m}, 1 \mathrm{H})$, $8.11(\mathrm{~d}, 1 \mathrm{H}, J=8.9 \mathrm{~Hz}), 7.93(\mathrm{~d}, 1 \mathrm{H}, J=8.2 \mathrm{~Hz}), 7.69(\mathrm{ddd}, 1 \mathrm{H}, J=8.1, J=6.9, J=1.2 \mathrm{~Hz}), 7.65(\mathrm{~d}, 2 \mathrm{H}$, $J=8.2 \mathrm{~Hz}), 7.60(\mathrm{ddd}, 1 \mathrm{H}, J=8.1, J=7.0, J=1.0 \mathrm{~Hz}), 7.50(\mathrm{~d}, 1 \mathrm{H}, J=8.6 \mathrm{~Hz}), 7.47(\mathrm{t}, 1 \mathrm{H}, J=8.2 \mathrm{~Hz})$; ${ }^{13}$ C-NMR (DMSO- $d_{6}$ ), $\delta: 169.90,160.22,136.15,134.14,132.10,130.02,129.37,128.69,127.58,126.12$, 124.56, 123.07, 122.72, 118.23, 106.37; HR-MS: [M - H] ${ }^{+}$calculated $330.00831 \mathrm{~m} / \mathrm{z}$, found $330.00943 \mathrm{~m} / \mathrm{z}$.

N-(3,4-Dichlorophenyl)-1-hydroxynaphthalene-2-carboxamide (12). Yield 84\%; m.p. 195-197 ${ }^{\circ} \mathrm{C}$; HPLC purity 99.14\%; IR ( $\left.\mathrm{cm}^{-1}\right)$ : 3429, 1628, 1601, 1584, 1574, 1527, 1504, 1467, 1390, 1320, 1298, 1274, 1247, 1210, 1144, 1127, 1088, 1026, 939, 871, 797, 785, 759, 729; ${ }^{1} \mathrm{H}-\mathrm{NMR}$ (DMSO-d ( $), \delta: 13.85$ (s, 1H), 10.76 (s, $1 \mathrm{H}), 8.28-8.33(\mathrm{~m}, 1 \mathrm{H}), 8.11(\mathrm{~d}, 1 \mathrm{H}, J=8.9 \mathrm{~Hz}), 7.93(\mathrm{~d}, 1 \mathrm{H}, J=8.2 \mathrm{~Hz}), 7.69(\mathrm{ddd}, 1 \mathrm{H}, J=8.1, J=6.9$, $J=1.2 \mathrm{~Hz}), 7.65(\mathrm{~d}, 2 \mathrm{H}, J=8.2 \mathrm{~Hz}), 7.60(\mathrm{ddd}, 1 \mathrm{H}, J=8.1, J=7.0, J=1.0 \mathrm{~Hz}), 7.50(\mathrm{~d}, 1 \mathrm{H}, J=8.6 \mathrm{~Hz})$, $7.47(\mathrm{t}, 1 \mathrm{H}, J=8.2 \mathrm{~Hz}) ;{ }^{13} \mathrm{C}-\mathrm{NMR}$ (DMSO- $\left.d_{6}\right), \delta: 169.56,159.90,137.90,136.06,130.86,130.52,129.28$, $127.47,126.21,126.00,124.56,123.09,123.00,122.94,121.68,117.99,107.42 ;$ HR-MS: [M - H] ${ }^{+}$calculated $330.00831 \mathrm{~m} / \mathrm{z}$, found $330.00970 \mathrm{~m} / \mathrm{z}$.

N-(3,5-Dichlorophenyl)-1-hydroxynaphthalene-2-carboxamide (13). Yield 80\%; m.p. 190-194 ${ }^{\circ} \mathrm{C}$; HPLC purity 99.09\%; IR ( $\left.\mathrm{cm}^{-1}\right)$ : 3431, 1626, 1585, 1568, 1526, 1504, 1443, 1407, 1387, 1300, 1267, 1204, 1144, 1086, 882, 823, 787, 755, 723; ${ }^{1} \mathrm{H}-\mathrm{NMR}$ (DMSO- $d_{6}$ ), $\delta: 13.48$ (br. s, $\left.1 \mathrm{H}\right), 10.61(\mathrm{~s}, 1 \mathrm{H}), 8.31(\mathrm{~d}, 1 \mathrm{H}$, $J=8.2 \mathrm{~Hz}), 8.04(\mathrm{~d}, 1 \mathrm{H}, J=8.9 \mathrm{~Hz}), 7.91(\mathrm{~d}, 1 \mathrm{H}, J=8.2 \mathrm{~Hz}), 7.88(\mathrm{~d}, 2 \mathrm{H}, J=1.6 \mathrm{~Hz}), 7.68(\mathrm{ddd}, 1 \mathrm{H}$, $J=8.1, J=7.0, J=1.3 \mathrm{~Hz}), 7.58(\mathrm{ddd}, 1 \mathrm{H}, J=8.1, J=7.0, J=1.0 \mathrm{~Hz}), 7.48(\mathrm{~d}, 1 \mathrm{H}, J=8.9 \mathrm{~Hz}), 7.39(\mathrm{t}$, $1 \mathrm{H}, J=1.8 \mathrm{~Hz}$ ); ${ }^{13} \mathrm{C}-\mathrm{NMR}$ (DMSO- $\left.d_{6}\right), \delta: 169.69,159.91,140.23,136.12,133.92,129.37,127.50,126.06$, 124.56, 123.70, 123.13, 122.94, 119.74, 118.08, 107.45; HR-MS: [M - H] ${ }^{+}$calculated $330.00831 \mathrm{~m} / \mathrm{z}$, found $330.00958 \mathrm{~m} / \mathrm{z}$.

1-Hydroxy-N-(2,4,5-trichlorophenyl)naphthalene-2-carboxamide (14). Yield 77\%; m.p. 211-215 ${ }^{\circ} \mathrm{C}$; HPLC purity $98.56 \%$; IR (cm $\left.{ }^{-1}\right)$ : 3422, 1630, 1574, 1514, 1501, 1470, 1446, 1392, 1367, 1320, 1287, 1269, 1242, 1205, 1146, 1088, 1068, 947, 884, 829, 876, 797, 785, 760, 715, 678; ${ }^{1} \mathrm{H}-\mathrm{NMR}$ (DMSO- $d_{6}$ ), $\delta: 13.39$ (br. s, $1 \mathrm{H}), 10.90(\mathrm{~s}, 1 \mathrm{H}), 8.33(\mathrm{~d}, 1 \mathrm{H}, J=8.7 \mathrm{~Hz}), 8.18(\mathrm{~s}, 1 \mathrm{H}), 8.06(\mathrm{~d}, 1 \mathrm{H}, J=8.7 \mathrm{~Hz}), 8.03(\mathrm{~s}, 1 \mathrm{H}), 7.93(\mathrm{~d}$, $1 \mathrm{H}, J=7.8 \mathrm{~Hz}), 7.68(\mathrm{ddd}, 1 \mathrm{H}, J=8.0, J=6.9, J=1.4 \mathrm{~Hz}), 7.60(\mathrm{ddd}, 1 \mathrm{H}, J=8.2, J=6.9, J=1.4 \mathrm{~Hz})$, $7.51(\mathrm{~d}, 1 \mathrm{H}, J=8.7 \mathrm{~Hz}) ;{ }^{13} \mathrm{C}-\mathrm{NMR}\left(\mathrm{DMSO}-d_{6}\right), \delta: 168.63,158.72,136.19,134.59,130.67,130.05,129.24$, 129.10, 128.55, 128.33, 127.64, 126.09, 124.71, 123.52, 123.16, 118.68, 108.30; HR-MS: [M - H] ${ }^{+}$calculated $363.96934 \mathrm{~m} / \mathrm{z}$, found $363.97119 \mathrm{~m} / \mathrm{z}$.

1-Hydroxy-N-(2,4,6-trichlorophenyl)naphthalene-2-carboxamide (15). Yield 75\%; m.p. 214-216 ${ }^{\circ} \mathrm{C}$; HPLC purity $98.91 \%$; IR $\left(\mathrm{cm}^{-1}\right)$ : 3303, 1631, 1616, 1597, 1556, 1524, 1500, 1468, 1445, 1407, 1385, 1320, 1278, 1241, 1211, 1189, 1154, 1142, 942, 851, 832, 796, 763, 713; ${ }^{1}$ H-NMR (DMSO-d ${ }_{6}$ ), $\delta: 13.73$ (br. s, $1 \mathrm{H}$ ), 10.79 (s, 1H), $8.30(\mathrm{~d}, 1 \mathrm{H}, J=8.7 \mathrm{~Hz}), 8.09(\mathrm{~d}, 1 \mathrm{H}, J=9.2 \mathrm{~Hz}), 7.93(\mathrm{~d}, 1 \mathrm{H}, J=8.2 \mathrm{~Hz}), 7.89(\mathrm{~s}, 2 \mathrm{H}), 7.70(\mathrm{ddd}$, $1 \mathrm{H}, J=8.2, J=6.9, J=1.4 \mathrm{~Hz}), 7.60(\mathrm{ddd}, 1 \mathrm{H}, J=8.2, J=7.1, J=1.1 \mathrm{~Hz}), 7.50(\mathrm{~d}, 1 \mathrm{H}, J=9.2 \mathrm{~Hz}) ;{ }^{13} \mathrm{C}-\mathrm{NMR}$ (DMSO- $\left.d_{6}\right), \delta: 169.92,160.22,136.19,134.96,133.31,131.54,129.46,128.50,127.61,126.18,124.53,123.09$, 122.68, 118.33, 106.27; HR-MS: [M - H] $]^{+}$calculated $363.96934 \mathrm{~m} / \mathrm{z}$, found $363.97119 \mathrm{~m} / \mathrm{z}$.

1-Hydroxy-N-(3,4,5-trichlorophenyl)naphthalene-2-carboxamide (16). Yield 71\%; m.p. 245-248 ${ }^{\circ} \mathrm{C}$; HPLC purity $99.28 \%$; IR ( $\left.\mathrm{cm}^{-1}\right)$ : 3430, 1627, 1598, 1575, 1515, 1501, 1467, 1432, 1380, 1356, 1309, 1287, 1267, 1244, 1205, 1150, 1076, 959, 882, 870, 822, 797, 787, 757, 723, 682; ${ }^{1} \mathrm{H}-\mathrm{NMR}$ (DMSO- $d_{6}$ ), $8: 13.41$ (br. s, $1 \mathrm{H}), 10.64(\mathrm{~s}, 1 \mathrm{H}), 8.29(\mathrm{~d}, 1 \mathrm{H}, J=8.2 \mathrm{~Hz}), 8.08(\mathrm{~s}, 2 \mathrm{H}), 8.02(\mathrm{~d}, 1 \mathrm{H}, J=9.2 \mathrm{~Hz}), 7.90(\mathrm{~d}, 1 \mathrm{H}, J=8.2 \mathrm{~Hz})$, 
7.65-7.70 (m, 1H), 7.55-7.60 (m, 1H), 7.47 (d, $1 \mathrm{H}, J=8.7 \mathrm{~Hz}) ;{ }^{13} \mathrm{C}-\mathrm{NMR}\left(\mathrm{DMSO}-d_{6}\right), \delta: 169.68,159.95$, 173.99, 136.14, 132.69, 129.42, 127.51, 126.08, 124.70, 124.55, 123.14, 122.86, 121.44, 118.12, 107.38; HR-MS: $[\mathrm{M}-\mathrm{H}]^{+}$calculated $363.96934 \mathrm{~m} / \mathrm{z}$, found $363.97119 \mathrm{~m} / \mathrm{z}$.

N-(2,4-Dibromophenyl)-1-hydroxynaphthalene-2-carboxamide (17). Yield 69\%; m.p. 185-188 ${ }^{\circ} \mathrm{C}$; HPLC purity $99.71 \%$; IR $\left(\mathrm{cm}^{-1}\right)$ : 3410, 1630, 1575, 1521, 1502, 1471, 1456, 1410, 1389, 1378, 1358, 1328, 1300, 1236, 1209, 1151, 1085, 943, 866, 819, 795, 786, 760, 718, 694; ${ }^{1}$ H-NMR (DMSO- $d_{6}$ ), $\delta: 13.72$ (br. s, $1 \mathrm{H}$ ), $10.69(\mathrm{~s}, 1 \mathrm{H}), 8.31(\mathrm{~d}, 1 \mathrm{H}, J=8.2 \mathrm{~Hz}), 8.07(\mathrm{~d}, 1 \mathrm{H}, J=8.7 \mathrm{~Hz}), 8.03(\mathrm{~d}, 1 \mathrm{H}, J=2.3 \mathrm{~Hz}), 7.93(\mathrm{~d}, 1 \mathrm{H}$, $J=7.8 \mathrm{~Hz}), 7.62-7.71(\mathrm{~m}, 3 \mathrm{H}) 7.57-7.62(\mathrm{~m}, 1 \mathrm{H}), 7.49(\mathrm{~d}, 1 \mathrm{H}, J=9.2 \mathrm{~Hz}) ;{ }^{13} \mathrm{C}-\mathrm{NMR}$ (DMSO- $\left.d_{6}\right), \delta: 169.22$, 159.45, 136.12, 135.28, 134.72, 131.31, 130.43, 129.23, 127.60, 126.05, 124.65, 123.16, 123.11, 121.83, 119.82, 118.34, 107.55; HR-MS: [M - H] $]^{+}$calculated $417.90727 \mathrm{~m} / z$, found $417.90927 \mathrm{~m} / z$.

N-(2,5-Dibromophenyl)-1-hydroxynaphthalene-2-carboxamide (18). Yield 72\%; m.p. 198-200 ${ }^{\circ} \mathrm{C}$; HPLC purity $98.82 \%$; IR ( $\left.\mathrm{cm}^{-1}\right)$ : 3393, 1642, 1630, 1576, 1522, 1502, 1467, 1401, 1390, 1357, 1323, 1284, 1237, 1206, 1150, 1086, 1027, 943, 898, 871, 788, 750, 725; ${ }^{1} \mathrm{H}-\mathrm{NMR}$ (DMSO-d $d_{6}$ ), $\delta: 13.58$ (br. s, $1 \mathrm{H}$ ), 10.78 (s, $1 \mathrm{H}), 8.33(\mathrm{~d}, 1 \mathrm{H}, J=8.2 \mathrm{~Hz}), 8.07(\mathrm{~d}, 1 \mathrm{H}, J=9.2 \mathrm{~Hz}), 7.99(\mathrm{~d}, 1 \mathrm{H}, J=2.3 \mathrm{~Hz}), 7.93(\mathrm{~d}, 1 \mathrm{H}, J=8.2 \mathrm{~Hz})$, $7.73(\mathrm{~d}, 1 \mathrm{H}, J=8.7 \mathrm{~Hz}), 7.66-7.71(\mathrm{~m}, 1 \mathrm{H}) 7.57-7.62(\mathrm{~m}, 1 \mathrm{H}), 7.47-7.52(\mathrm{~m}, 2 \mathrm{H}) ;{ }^{13} \mathrm{C}-\mathrm{NMR}\left(\right.$ DMSO- $\left.d_{6}\right), \delta$ : 168.98, 159.15, 137.32, 136.14, 134.34, 131.02, 130.97, 129.22, 127.62, 126.06, 124.68, 123.32, 123.13, 120.42, 119.44, 118.46, 107.87; HR-MS: [M - H] ${ }^{+}$calculated $417.90727 \mathrm{~m} / \mathrm{z}$, found $417.90927 \mathrm{~m} / \mathrm{z}$.

N-(2,6-Dibromophenyl)-1-hydroxynaphthalene-2-carboxamide (19). Yield 64\%; m.p. 212-216 ${ }^{\circ} \mathrm{C}$; HPLC purity $98.36 \%$; IR $\left(\mathrm{cm}^{-1}\right)$ : 3283, 1633, 1616, 1595, 1558, 1522, 1498, 1465, 1441, 1429, 1408, 1382, 1325, 1281, 1209, 1191, 1152, 944, 793, 763, 720, 697; ${ }^{1} \mathrm{H}-\mathrm{NMR}$ (DMSO-d $\left.d_{6}\right), \delta: 13.89$ (s, 1H), $10.81(\mathrm{~s}, 1 \mathrm{H}), 8.30$ $(\mathrm{d}, 1 \mathrm{H}, J=8.6 \mathrm{~Hz}), 8.11(\mathrm{~d}, 1 \mathrm{H}, J=8.9 \mathrm{~Hz}), 7.93(\mathrm{~d}, 1 \mathrm{H}, J=8.2 \mathrm{~Hz}), 7.83(\mathrm{~d}, 2 \mathrm{H}, J=8.2 \mathrm{~Hz}), 7.69(\mathrm{ddd}$, $1 \mathrm{H}, J=8.1, J=6.9, J=1.2 \mathrm{~Hz}), 7.60(\mathrm{ddd}, 1 \mathrm{H}, J=8.1, J=7.0, J=1.0 \mathrm{~Hz}), 7.50(\mathrm{~d}, 1 \mathrm{H}, J=8.9 \mathrm{~Hz}), 7.30(\mathrm{t}$, $1 \mathrm{H}, J=8.1 \mathrm{~Hz}$ ); ${ }^{13} \mathrm{C}-\mathrm{NMR}$ (DMSO- $\left.d_{6}\right), \delta: 169.70,160.19,136.12,134.85,132.34,130.87,129.31,127.55$, 126.08, 124.59, 124.55, 123.03, 122.72, 118.16, 106.46; HR-MS: $[\mathrm{M}-\mathrm{H}]^{+}$calculated $417.9727 \mathrm{~m} / \mathrm{z}$, found $417.90894 \mathrm{~m} / \mathrm{z}$.

1-Hydroxy-N-(2,4,6-tribromophenyl)naphthalene-2-carboxamide (20). Yield 77\%; m.p. 115-118 ${ }^{\circ} \mathrm{C}$; HPLC purity $99.18 \%$; IR $\left(\mathrm{cm}^{-1}\right)$ : 3266, 1610, 1596, 1520, 1500, 1467, 1440, 1387, 1371, 1321, 1273, 1256, 1209, 1153, 1089, 943, 854, 805, 793, 764, 744, 721, 697; ${ }^{1} \mathrm{H}-\mathrm{NMR}$ (DMSO-d $d_{6}$ ), $8: 13.79$ (br. s, $1 \mathrm{H}$ ), 10.81 (s, $1 \mathrm{H}), 8.30(\mathrm{~d}, 1 \mathrm{H}, J=8.7 \mathrm{~Hz}), 8.13(\mathrm{~s}, 2 \mathrm{H}), 8.08(\mathrm{~d}, 1 \mathrm{H}, J=8.7 \mathrm{~Hz}), 7.93(\mathrm{~d}, 1 \mathrm{H}, J=8.2 \mathrm{~Hz}), 7.67-7.72(\mathrm{~m}$, 1H), 7.58-7.63 (m, 1H), 7.50 (d, $1 \mathrm{H}, J=8.7 \mathrm{~Hz}) ;{ }^{13} \mathrm{C}-\mathrm{NMR}$ (DMSO- $\left.d_{6}\right), \delta: 169.62,160.25,136.17,134.71$, $134.48,129.42,127.60,126.16,125.44,124.55,123.07,122.69,121.88,118.26,106.37 ;$ HR-MS: $[\mathrm{M}-\mathrm{H}]^{+}$ calculated $495.81777 \mathrm{~m} / \mathrm{z}$, found $495.82037 \mathrm{~m} / \mathrm{z}$.

$\mathrm{N}$-(2-Bromo-4-chlorophenyl)-1-hydroxynaphthalene-2-carboxamide (21). Yield 69\%; m.p. 182-184 ${ }^{\circ} \mathrm{C}$; HPLC purity $99.02 \%$; IR $\left(\mathrm{cm}^{-1}\right)$ : 3404, 1631, 1575, 1520, 1503, 1471, 1453, 1414, 1387, 1328, 1296, 1271, 1234, 1208, 1140, 1087, 1033, 1023, 941, 858, 832, 822, 796, 788, 758, 733, 706, 694; ${ }^{1}$ H-NMR (DMSO- $d_{6}$ ), $\delta:$ $13.72(\mathrm{~s}, 1 \mathrm{H}), 10.71(\mathrm{~s}, 1 \mathrm{H}), 8.31(\mathrm{~d}, J=8.3 \mathrm{~Hz}, 1 \mathrm{H}), 8.07(\mathrm{~d}, J=8.8 \mathrm{~Hz}, 1 \mathrm{H}), 7.91-7.94(\mathrm{~m}, 2 \mathrm{H}), 7.70(\mathrm{~d}$, $J=8.6 \mathrm{~Hz}, 1 \mathrm{H}), 7.66-7.70(\mathrm{~m}, 1 \mathrm{H}), 7.55-7.62(\mathrm{~m}, 2 \mathrm{H}), 7.49(\mathrm{~d}, J=8.8 \mathrm{~Hz}, 1 \mathrm{H}) ;{ }^{13} \mathrm{C}-\mathrm{NMR}\left(\right.$ DMSO- $\left.d_{6}\right), \delta$ : 169.30, 159.48, 136.10, 134.88, 132.04, 131.77, 130.12, 129.21, 128.37, 127.58, 126.04, 124.65, 123.13, 123.10, 121.59, 118.32, 107.49; HR-MS: [M + H] $]^{+}$calculated $375.97344 \mathrm{~m} / \mathrm{z}$, found $375.97415 \mathrm{~m} / \mathrm{z}$.

N-(4-Bromo-2-chlorophenyl)-1-hydroxynaphthalene-2-carboxamide (22). Yield 56\%; m.p. $186-187^{\circ} \mathrm{C}$; HPLC purity $98.84 \%$; IR $\left(\mathrm{cm}^{-1}\right)$ : 3425, 1628, 1588, 1575, 1520, 1502, 1471, 1456, 1409, 1390, 1382, 1359, 1327, 1299, 1236, 1210, 1168, 1149, 1078, 1048, 1022, 943, 868, 811, 796, 786, 760, 735, 711, 698; ${ }^{1} \mathrm{H}-\mathrm{NMR}$ (DMSO- $\left.d_{6}\right), \delta: 13.63(\mathrm{~s}, 1 \mathrm{H}), 10.73(\mathrm{~s}, 1 \mathrm{H}), 8.32(\mathrm{~d}, J=8.3 \mathrm{~Hz}, 1 \mathrm{H}), 8.07(\mathrm{~d}, J=9.1 \mathrm{~Hz}, 1 \mathrm{H}), 7.93(\mathrm{~d}$, $J=8.3 \mathrm{~Hz}, 1 \mathrm{H}), 7.90(\mathrm{~d}, J=2.0 \mathrm{~Hz}, 1 \mathrm{H}), 7.63-7.71(\mathrm{~m}, 3 \mathrm{H}), 7.57-7.62(\mathrm{~m}, 1 \mathrm{H}), 7.49(\mathrm{~d}, J=8.8 \mathrm{~Hz}, 1 \mathrm{H})$; ${ }^{13}$ C-NMR (DMSO- $\left.d_{6}\right), \delta: 169.06,159.23,136.12,133.81,131.85,130.79,130.77,129.89,129.19,127.60$, 126.04, 124.67, 123.27, 123.11, 119.30, 118.42, 107.76; HR-MS: $[\mathrm{M}+\mathrm{H}]^{+}$calculated $375.97344 \mathrm{~m} / \mathrm{z}$, found $375.97415 \mathrm{~m} / \mathrm{z}$. 
N-(5-Bromo-2-chlorophenyl)-1-hydroxynaphthalene-2-carboxamide (23). Yield 35\%; m.p. 178-180 ${ }^{\circ} \mathrm{C} ; \mathrm{HPLC}$ purity 99.26\%; IR ( $\left.\mathrm{cm}^{-1}\right)$ : 3422, 1632, 1582, 1524, 1503, 1470, 1404, 1388, 1360, 1323, 1288, 1271, 1260, 1240, 1207, 1165, 1151, 1087, 1080, 1041, 1033, 942, 866, 796, 784, 755, 727, 715, 705; ${ }^{1} \mathrm{H}-\mathrm{NMR}\left(\mathrm{DMSO}-\mathrm{d}_{6}\right)$, $\delta: 13.48$ (br. s, 1H), $10.82(\mathrm{~s}, 1 \mathrm{H}), 8.33(\mathrm{~d}, J=8.3 \mathrm{~Hz}, 1 \mathrm{H}), 8.07$ (d, J = 7.3 Hz, 1H), 8.05 (s, $1 \mathrm{H}), 7.93(\mathrm{~d}$, $J=8.1 \mathrm{~Hz}, 1 \mathrm{H}), 7.66-7.71(\mathrm{~m}, 1 \mathrm{H}), 7.53-7.63(\mathrm{~m}, 3 \mathrm{H}), 7.51(\mathrm{~d}, J=8.8 \mathrm{~Hz}, 1 \mathrm{H}) ;{ }^{13} \mathrm{C}-\mathrm{NMR}\left(\mathrm{DMSO}-d_{6}\right), \delta$ : 168.76, 158.86, 136.16, 135.82, 131.23, 130.38, 130.33, 129.19, 128.40, 127.63, 126.07, 124.71, 123.47, 123.15, 119.72, 118.59, 108.18; HR-MS: $[\mathrm{M}+\mathrm{H}]^{+}$calculated $375.97344 \mathrm{~m} / \mathrm{z}$, found $375.97421 \mathrm{~m} / \mathrm{z}$.

N-(4-Bromo-3-chlorophenyl)-1-hydroxynaphthalene-2-carboxamide (24). Yield 58\%; m.p. 203-205 ${ }^{\circ} \mathrm{C}$; HPLC purity 99.53\%; IR ( $\left.\mathrm{cm}^{-1}\right)$ : 3429, 1627, 1599, 1572, 1520, 1504, 1464, 1406, 1381, 1321, 1293, 1272, 1245, $1207,1150,1139,1114,1086,1012,935,869,794,783,757,727,695 ;{ }^{1} \mathrm{H}-\mathrm{NMR}$ (DMSO-d $), \delta: 13.61$ (s, $\left.1 \mathrm{H}\right)$, $10.67(\mathrm{~s}, 1 \mathrm{H}), 8.31(\mathrm{~d}, J=8.3 \mathrm{~Hz}, 1 \mathrm{H}), 8.12(\mathrm{~d}, J=2.3 \mathrm{~Hz}, 1 \mathrm{H}), 8.07(\mathrm{~d}, J=9.1 \mathrm{~Hz}, 1 \mathrm{H}), 7.91(\mathrm{~d}, J=8.1 \mathrm{~Hz}$, $1 \mathrm{H}), 7.80(\mathrm{~d}, J=8.8 \mathrm{~Hz}, 1 \mathrm{H}), 7.66-7.72(\mathrm{~m}, 2 \mathrm{H}), 7.56-7.61(\mathrm{~m}, 1 \mathrm{H}), 7.48(\mathrm{~d}, J=8.8 \mathrm{~Hz}, 1 \mathrm{H}) ;{ }^{13} \mathrm{C}-\mathrm{NMR}$ (DMSO- $\left.d_{6}\right), \delta: 169.58,159.92,138.51,136.08,133.76,132.92,129.32,127.50,126.04,124.58,123.10,122.96$, 122.89, 121.83, 118.01, 116.08, 107.45; HR-MS: $[\mathrm{M}+\mathrm{H}]^{+}$calculated $375.97344 \mathrm{~m} / \mathrm{z}$, found $375.97397 \mathrm{~m} / \mathrm{z}$.

\subsection{Study of Inhibition of Photosynthetic Electron Transport (PET) in Spinach Chloroplasts}

Chloroplasts were prepared from spinach (Spinacia oleracea L.) according to Masarovicova and Kralova [49]. The inhibition of photosynthetic electron transport (PET) in spinach chloroplasts was determined spectrophotometrically (Genesys 6, Thermo Scientific, USA), using an artificial electron acceptor 2,6-dichlorophenol-indophenol (DCIPP) according to Kralova et al. [50], and the rate of photosynthetic electron transport was monitored as photoreduction of DCPIP. The measurements were carried out in phosphate buffer $(0.02 \mathrm{M}, \mathrm{pH} 7.2)$ containing sucrose $(0.4 \mathrm{M}), \mathrm{MgCl}_{2}(0.005 \mathrm{M})$ and $\mathrm{NaCl}(0.015 \mathrm{M})$. The chlorophyll content was $30 \mathrm{mg} / \mathrm{L}$ in these experiments, and the samples were irradiated $\left(\sim 100 \mathrm{~W} / \mathrm{m}^{2}\right.$ with $10 \mathrm{~cm}$ distance) with a halogen lamp $(250 \mathrm{~W})$ using a 4-cm water filter to prevent warming of the samples (suspension temperature $22{ }^{\circ} \mathrm{C}$ ). The studied compounds were dissolved in DMSO due to their limited water solubility. The applied DMSO concentration (up to 4\%) practically did not affect the photochemical activity in spinach chloroplasts. The inhibitory efficiency of the studied compounds was expressed by $\mathrm{IC}_{50}$ values, i.e., by molar concentration of the compounds causing a $50 \%$ decrease in the oxygen evolution rate relative to the untreated control. The comparable $\mathrm{IC}_{50}$ value for a selective herbicide 3-(3,4-dichlorophenyl)-1,1-dimethylurea, DCMU (Diuron ${ }^{\circledR}$, Merck, Darmstadt, Germany) was about $1.9 \mu \mathrm{M}$. The results are summarized in Table 1.

\subsection{Study of Fluorescence of Chlorophyll a and Aromatic Amino Acids in Spinach Chloroplasts}

The fluorescence emission spectra of chlorophyll $a(\mathrm{Chl} a)$ and aromatic amino acids in spinach chloroplasts were recorded on fluorescence spectrophotometer F-2000 (Hitachi, Tokyo, Japan) using excitation wavelength $\lambda_{\mathrm{ex}}=436 \mathrm{~nm}$ for monitoring the fluorescence of Chla and $\lambda_{\mathrm{ex}}=275 \mathrm{~nm}$ for monitoring the fluorescence of aromatic amino acids, excitation slit $20 \mathrm{~nm}$ and emission slit $10 \mathrm{~nm}$. The samples were kept in the dark for 2 min prior to the measurement. The phosphate buffer used for dilution of the chloroplast suspension was the same as described above. Due to low aqueous solubility, the compounds were added to a chloroplast suspension in DMSO solution. The DMSO concentration in all samples was the same as in the control (10\%). The chlorophyll concentration in chloroplast suspension was $10 \mathrm{mg} / \mathrm{L}$.

\section{Conclusions}

Series of seventeen new di- and trichlorinated and brominated 1-hydroxynaphthalene2-carboxanilides was prepared and characterized. These compounds were completed by seven recently published unsubstituted and monochlorinated and brominated derivatives, and all the compounds were tested for their in vitro activity related to the inhibition of photosynthetic electron transport (PET) in spinach (Spinacia oleracea L.) chloroplasts. N-(3,5-dichlorophenyl)-(13), 
$\mathrm{N}$-(4-bromo-3-chlorophenyl)-(24), $\mathrm{N}$-(2,5-dibromophenyl)-(18) and 1-hydroxy- $N$-(3,4,5-trichlorophenyl)naphthalene-2-carboxamide (16) were the most potent PET inhibitors with $\mathrm{IC}_{50}=5.2,6.7$, 7.6 and $8.0 \mu \mathrm{M}$, respectively. In general, it can be stated that the chlorinated derivatives demonstrated higher potency than the bromine derivatives. The inhibitory activity of these compounds depends on the position and the type of halogen substituents, e.g., all 2,4-disubstituted compounds 9, 17, 21, 22 and 2,3-disubstituted compound $\mathbf{8}$ showed limited aqueous solubility resulting in moderate PET-inhibiting activity compared with that of 2,5-, 2,6-, 3,4- or 3,5-disubstituted derivatives or other investigated compounds. It can be stated that the PET inhibition increases with increasing lipophilicity for monoand disubstituted analogues, while an opposite trend is observed for trisubstituted derivatives. Thus, it seems that for PET-inhibiting activity, a lipophilicity optimum is in the range of $\log P$ from 6.01 to 6.28. On the other hand, the PET inhibition decreases with increasing electron-withdrawing properties of individual anilides, and this trend is similar for all the series. Interactions of the studied compounds with chlorophyll $a$ and aromatic amino acids present in pigment-protein complexes mainly in PS II were documented by fluorescence spectroscopy. The section between $\mathrm{P}_{680}$ and plastoquinone $\mathrm{Q}_{\mathrm{B}}$ in the PET chain occurring on the acceptor side of PS II can be suggested as the site of action of the compounds.

Acknowledgments: This study was supported by the Slovak Research and Development Agency (Grant No. APVV-0516-12) and VEGA 1/0770/15. The HPLC/HRMS system forms a part of the National Infrastructure CzeCOS ProCES CZ.02.1.01/0.0/0.0/16_013/0001609; Michal Oravec was supported by the National Sustainability Program (NPU I; Grant No. LO1415).

Author Contributions: Tomas Gonec, Jiri Kos, Josef Jampilek: design, synthesis of the compounds, SAR, writing of the paper. Michal Oravec, Jana Dohanosova, Tibor Liptaj: analysis/characterization of the compounds. Matus Pesko and Katarina Kralova: PET evaluation.

Conflicts of Interest: The authors declare no conflicts of interest.

\section{References}

1. Draber, W.; Tietjen, K.; Kluth, J.F.; Trebst, A. Herbicides in photosynthesis research. Angew. Chem. 1991, 3, 1621-1633. [CrossRef]

2. Tischer, W.; Strotmann, H. Relationship between inhibitor binding by chloroplasts and inhibition of photosynthetic electron-transport. Biochim. Biophys. Acta 1977, 460, 113-125. [CrossRef]

3. Trebst, A.; Draber, W. Structure activity correlations of recent herbicides in photosynthetic reactions. In Advances in Pesticide Science; Greissbuehler, H., Ed.; Pergamon Press: Oxford, UK, 1979; pp. 223-234.

4. Bowyer, J.R.; Camilleri, P.; Vermaas, W.F.J. Herbicides, Topics in Photosynthesis; Baker, N.R., Percival, M.P., Eds.; Elsevier: Amsterdam, The Netherlands, 1991; pp. 27-85.

5. Izawa, S. Acceptors and donors for chloroplast electron transport. In Methods in Enzymology; Part C; Colowick, P., Kaplan, N.O., Eds.; Academic Press: London, UK, 1980; pp. 413-434.

6. Whitmarsh, J. Electron transport and energy transduction. In Photosynthesis: A Comprehensive Treatise; Raghavendra, A.S., Ed.; Cambridge University Press: Cambridge, UK, 1998; pp. 87-110.

7. Huppatz, J.L.; McFadden, H.G. Understanding the topography of the photosystem II herbicide binding niche: Does QSAR help? Z. Naturforsch. 1993, 48, 140-145.

8. Lambreva, M.D.; Russo, D.; Polticelli, F.; Scognamiglio, V.; Antonacci, A.; Zobnina, V.; Campi, G.; Rea, G. Structure/function/dynamics of photosystem II plastoquinone binding sites. Curr. Protein Pept. Sci. 2014, 15, 285-295. [CrossRef] [PubMed]

9. Trebst, A. Inhibitors in the functional dissection of the photosynthetic electron transport system. Photosynth Res. 2007, 92, 217-224. [CrossRef] [PubMed]

10. Teixeira, R.R.; de Andrade Barros, M.V.; Bressan, G.C.; Siqueira, R.P.; Dos Santos, F.S.; Bertazzini, M.; Kiralj, R.; Ferreira, M.M.C.; Forlani, G. Synthesis, theoretical studies, and effect on the photosynthetic electron transport of trifluoromethyl arylamides. Pest Manag. Sci. 2017, in press. [CrossRef] [PubMed]

11. Gonec, T.; Kos, J.; Zadrazilova, I.; Pesko, M.; Keltosova, S.; Tengler, J.; Bobal, P.; Kollar, P.; Cizek, A.; Kralova, K.; et al. Antimycobacterial and herbicidal activity of ring-substituted 1-hydroxynaphthalene2-carboxanilides. Bioorg. Med. Chem. 2013, 21, 6531-6541. [CrossRef] [PubMed] 
12. Kralova, K.; Perina, M.; Waisser, K.; Jampilek, J. Structure-activity relationships of N-benzylsalicylamides for inhibition of photosynthetic electron transport. Med. Chem. 2015, 11, 156-164. [CrossRef] [PubMed]

13. Imramovsky, A.; Pesko, M.; Monreal-Ferriz, J.; Kralova, K.; Vinsova, J.; Jampilek, J. Photosynthesis-inhibiting efficiency of 4-chloro-2-(chlorophenylcarbamoyl)phenyl alkyl-carbamates. Bioorg. Med. Chem. Lett. 2011, 21, 4564-4567. [CrossRef] [PubMed]

14. Pattabiraman, V.R.; Bode, J.W. Rethinking amide bond synthesis. Nature 2011, 480, 471-479. [CrossRef] [PubMed]

15. Ghosh, A.K.; Brindisi, M. Organic carbamates in drug design and medicinal chemistry. J. Med. Chem. 2015, 58, 2895-2940. [CrossRef] [PubMed]

16. Gonec, T.; Zadrazilova, I.; Nevin, E.; Kauerova, T.; Pesko, M.; Kos, J.; Oravec, M.; Kollar, P.; Coffey, A.; O'Mahony, J.; et al. Synthesis and biological evaluation of $\mathrm{N}$-alkoxyphenyl-3-hydroxynaphthalene-2carboxanilides. Molecules 2015, 20, 9767-9787. [CrossRef] [PubMed]

17. Teixeira, R.R.; Pereira, J.L.; Pereira, W.L. Photosynthetic inhibitors. In Applied Photosynthesis; Najafpour, M., Ed.; InTech: Rijeka, Croatia, 2012; pp. 3-22.

18. Dolezal, M.; Zitko, J.; Osicka, Z.; Kunes, J.; Vejsova, M.; Buchta, V.; Dohnal, J.; Jampilek, J.; Kralova, K. Synthesis, antimycobacterial, antifungal and photosynthesis-inhibiting activity of chlorinated $\mathrm{N}$-phenylpyrazine-2-carboxamides. Molecules 2010, 15, 8567-8581. [CrossRef] [PubMed]

19. Imramovsky, A.; Pesko, M.; Kralova, K.; Vejsova, M.; Stolarikova, J.; Vinsova, J.; Jampilek, J. Investigating spectrum of biological activity of 4 - and 5-chloro-2-hydroxy- $N$-[2-(arylamino)-1-alkyl-2-oxoethyl]benzamides. Molecules 2011, 16, 2414-2430. [CrossRef] [PubMed]

20. Gonec, T.; Bobal, P.; Sujan, J.; Pesko, M.; Guo, J.; Kralova, K.; Pavlacka, L.; Vesely, L.; Kreckova, E.; Kos, J.; et al. Investigating the spectrum of biological activity of substituted quinoline-2-caboxamides and their isosteres. Molecules 2012, 17, 613-644. [CrossRef] [PubMed]

21. Kos, J.; Zadrazilova, I.; Pesko, M.; Keltosova, S.; Tengler, J.; Gonec, T.; Bobal, P.; Kauerova, T.; Oravec, M.; Kollar, P.; et al. Antibacterial and herbicidal activity of ring-substituted 3-hydroxynaphthalene2-carboxanilides. Molecules 2013, 18, 7977-7997. [CrossRef] [PubMed]

22. Gonec, T.; Kos, J.; Zadrazilova, I.; Pesko, M.; Govender, R.; Keltosova, S.; Chambel, B.; Pereira, D.; Kollar, P.; Imramovsky, A.; et al. Antibacterial and herbicidal activity of ring-substituted 2-hydroxynaphthalene1-carboxanilides. Molecules 2013, 18, 9397-9419. [CrossRef] [PubMed]

23. Jampilek, J.; Kralova, K.; Pesko, M.; Kos, J. Ring-substituted 8-hydroxyquinoline-2-carboxanilides as photosystem II inhibitors. Bioorg. Med. Chem. Lett. 2016, 26, 3862-3865.

24. Fajkusova, D.; Pesko, M.; Keltosova, S.; Guo, J.; Oktabec, Z.; Vejsova, M.; Kollar, P.; Coffey, A.; Csollei, J.; Kralova, K.; et al. Anti-infective and herbicidal activity of $N$-substituted 2-aminobenzothiazoles. Bioorg. Med. Chem. 2012, 20, 7059-7068. [CrossRef] [PubMed]

25. Pauk, K.; Zadrazilova, I.; Imramovsky, A.; Vinsova, J.; Pokorna, M.; Masarikova, M.; Cizek, A.; Jampilek, J. New derivatives of salicylamides: Preparation and antimicrobial activity against various bacterial species. Bioorg. Med. Chem. 2013, 21, 6574-6581. [CrossRef] [PubMed]

26. Kos, J.; Nevin, E.; Soral, M.; Kushkevych, I.; Gonec, T.; Bobal, P.; Kollar, P.; Coffey, A.; O’Mahony, J.; Liptaj, T.; et al. Synthesis and antimycobacterial properties of ring-substituted 6-hydroxynaphthalene2-carboxanilides. Bioorg. Med. Chem. 2015, 23, 2035-2043. [CrossRef] [PubMed]

27. Kos, J.; Zadrazilova, I.; Nevin, E.; Soral, M.; Gonec, T.; Kollar, P.; Oravec, M.; Coffey, A.; O’Mahony, J.; Liptaj, T.; et al. Ring-Substituted 8-hydroxyquinoline-2-carboxanilides as potential antimycobacterial agents. Bioorg. Med. Chem. 2015, 23, 4188-4196. [CrossRef] [PubMed]

28. Gonec, T.; Kralova, K.; Pesko, M.; Jampilek, J. Antimycobacterial N-alkoxyphenylhydroxynaphthalenecarboxamides affecting photosystem II. Bioorg. Med. Chem. Lett. 2017, 27, 1881-1885. [CrossRef] [PubMed]

29. Otevrel, J.; Mandelova, Z.; Pesko, M.; Guo, J.; Kralova, K.; Sersen, F.; Vejsova, M.; Kalinowski, D.; Kovacevic, Z.; Coffey, A.; et al. Investigating the spectrum of biological activity of ring-substituted salicylanilides and carbamoylphenylcarbamates. Molecules 2010, 15, 8122-8142. [CrossRef] [PubMed]

30. Zadrazilova, I.; Pospisilova, S.; Masarikova, M.; Imramovsky, A.; Monreal-Ferriz, J.; Vinsova, J.; Cizek, A.; Jampilek, J. Salicylanilide carbamates: Promising antibacterial agents with high in vitro activity against methicillin-resistant Staphylococcus aureus. Eur. J. Pharm. Sci. 2015, 77, 197-207. [CrossRef] [PubMed]

31. Gonec, T.; Pospisilova, S.; Kauerova, T.; Kos, J.; Dohanosova, J.; Oravec, M.; Kollar, P.; Coffey, A.; Liptaj, T.; Cizek, A.; et al. N-Alkoxyphenylhydroxynaphthalenecarboxamides and their antimycobacterial activity. Molecules 2016, 21, 1068. [CrossRef] [PubMed] 
32. Gonec, T.; Pospisilova, S.; Holanova, L.; Stranik, J.; Cernikova, A.; Pudelkova, V.; Kos, J.; Oravec, M.; Kollar, P.; Cizek, A.; et al. Synthesis and antimicrobial evaluation of 1-[(2-substituted phenyl)carbamoyl]naphthalen2-yl carbamates. Molecules 2016, 21, 1189. [CrossRef] [PubMed]

33. Gonec, T.; Stranik, J.; Pesko, M.; Kos, J.; Oravec, M.; Kralova, K.; Jampilek, J. Photosynthesis-inhibiting activity of 1-[(2-chlorophenyl)carbamoyl]- and 1-[(2-nitrophenyl)carbamoyl]naphthalen-2-yl alkylcarbamates. Molecules 2017, 22, 1199. [CrossRef] [PubMed]

34. Vettorazzi, M.; Angelina, E.; Lima, S.; Gonec, T.; Otevrel, J.; Marvanova, P.; Padrtová, T.; Mokry, P.; Bobal, P.; Acosta, L.M.; et al. Search of new structural scaffolds for sphingosine kinase 1 inhibitors. Eur. J. Med. Chem. 2017, 139, 461-481. [CrossRef] [PubMed]

35. Jampilek, J. Potential of agricultural fungicides for antifungal drug discovery. Expert Opin. Drug Dis. 2016, 11, 1-9. [CrossRef] [PubMed]

36. Xiao, R.; Ghosh, S.; Tanaka, A.R.; Greenberg, B.M.; Dumbroff, E.B. A rapid spectrophotometric method for measuring photosystem I and photosystem II in a single sample. Plant Physiol. Biochem. 1997, 35, 411-417.

37. Sersen, F.; Kralova, K.; Macho, V. New findings about the inhibitory action of phenylcarbamates and phenylthiocarbamates on photosynthetic apparatus. Pest. Biochem. Physiol. 2000, 68, 113-118. [CrossRef]

38. Rashid, A.; Popovic, R. Electron donation to photosystem II by diphenylcarbazide is inhibited both by the endogenous manganese complex and by exogenous manganese ions. Biochem. Cell. Biol. 1995, 73, 241-245. [CrossRef] [PubMed]

39. Jegerschold, C.; Styring, S. Fast oxygen-independent degradation of the Dl reaction centre protein in photosystem II. FEBS Lett. 1991, 280, 87-90. [CrossRef]

40. Purcell, M.; Leroux, G.; Carpentier, R. Interaction of the electron donor diphenylcarbazide with the herbicide-binding niche of photosystem II. Biochim. Biophys. Acta Int. J. Biochem. Biophys. 1991, 1058, 374-378. [CrossRef]

41. Borse, T.H.; Maheswarim, V.L.; Baviskar, M.P. Effect of diphenyl carbazide on the metribuzin induced inhibition of photosystem-II photochemistry. J. Plant Biochem. Biotechn. 2000, 9, 119-121. [CrossRef]

42. Servusova, B.; Eibinova, D.; Dolezal, M.; Kubicek, V.; Paterova, P.; Pesko, M.; Kralova, K. Substituted N-benzylpyrazine-2-carboxamides: Synthesis and biological evaluation. Molecules 2012, 17, 13183-13198. [CrossRef] [PubMed]

43. Govindjee, A. Sixty-three years since Kautsky: Chlorophyll a fluorescence. Aust. J. Plant Physiol. 1995, 22, 131-160. [CrossRef]

44. Kralova, K.; Sersen, F.; Kubicova, L.; Waisser, K. Inhibitory effects of substituted benzanilides on photosynthetic electron transport in spinach chloroplasts. Chem. Pap. 1999, 53, 328-331. [CrossRef]

45. Kralova, K.; Sersen, F.; Pesko, M.; Waisser, K.; Kubicova, L. 5-Bromo- and 3,5-dibromo-2-hydroxy$\mathrm{N}$-phenylbenzamides-Inhibitors of photosynthesis. Chem. Pap. 2014, 68, 46-52. [CrossRef]

46. Kralova, K.; Sersen, F.; Miletin, M.; Hartl, J. Inhibition of photosynthetic electron transport by some anilides of 2-alkylpyridine-4-carboxylic acids in spinach chloroplasts. Chem. Pap. 1998, 52, 52-58. [CrossRef]

47. Jandourek, O.; Dolezal, M.; Paterova, P.; Kubicek, V.; Pesko, M.; Kunes, J.; Coffey, A.; Guo, J.; Kralova, K. $\mathrm{N}$-Substituted 5-amino-6-methylpyrazine-2,3-dicarbonitriles: Microwave-assisted synthesis and biological properties. Molecules 2014, 19, 651-671. [CrossRef] [PubMed]

48. Imramovsky, A.; Pesko, M.; Jampilek, J.; Kralova, K. Synthesis and photosynthetic electron transport inhibition of 2-substituted 6-fluorobenzothiazoles. Monatsh. Chem. 2014, 145, 1817-1824. [CrossRef]

49. Masarovicova, E.; Kralova, K. Approaches to measuring plant photosynthesis activity. In Handbook of Photosynthesis, 2nd ed.; Pessarakli, M., Ed.; Taylor \& Francis Group: Boca Raton, FL, USA, 2005; pp. 617-656.

50. Kralova, K.; Sersen, F.; Sidoova, E. Photosynthesis inhibition produced by 2-alkylthio-6-R-benzothiazoles. Chem. Pap. 1992, 46, 348-350.

Sample Availability: Samples of compounds 1-24 are available from author T. Gonec. 